\title{
MINIMAL COMMUTATIVE DIVISIBLE SEMIGROUPS
}

\author{
BY TAKAYUKI TAMURA
}

Communicated by Edwin Hewitt, May 22, 1963

1. Introduction. Let $S$ be a commutative divisible semigroup whose binary operation is denoted by + . If for any element $x$ of $S$ and for any positive integer $n$, there is an element $y \in S$ such that

$$
x=n \cdot y=y+\cdots+y, \quad n \text { times }
$$

then $S$ is said to be divisible. If $y$ is unique then $S$ is called uniquely divisible. For example the additive semigroup $R$ of all positive rational numbers is a uniquely divisible semigroup, while the additive group $R /(1)$ of all positive rational numbers mod 1 is divisible but not uniquely divisible.

In this note we report some results on commutative divisible semigroups, especially minimal commutative divisible semigroups, without proof. The proof of the theorems will be given in another paper [6]. Throughout this paper any semigroup is assumed to be commutative, and "commutative" will be often omitted.

2. Fundamental theorems. The following basic propositions are used for the discussion in this paper.

(2.1) Any homomorphic image of a divisible semigroup is divisible.

(2.2) If $S_{i}(i=1, \cdots, k)$ are divisible (uniquely divisible) then the direct product of $S_{i}$ is also divisible (uniquely divisible).

Suppose a system of divisible semigroups $S_{\alpha}(\alpha \in \Lambda)$ is given. $S_{\alpha}^{0}$ denotes the semigroup $S_{\alpha}$ with a two-sided identity 0 adjoined: $x+0=0+x=x$ for all $x \in S_{\alpha}$. The semigroup obtained as the discrete direct product $\prod_{\alpha \in \Delta} S_{\alpha}^{0}$ excluding the identity is called the annexed product of $S_{\alpha}$, and it is denoted by $\tilde{\Pi} \tilde{\Pi}_{\alpha \in \Lambda} S_{\alpha}$.

(2.3) If $S_{\alpha}$ is divisible (uniquely divisible) then $\tilde{\Pi}_{\alpha \in \Lambda} S_{\alpha}$ is also divisible (uniquely divisible).

A commutative semigroup $S$ is called power-cancellative if $n \cdot x$ $=n \cdot y$ implies $x=y$ for every $n$.

(2.4) Any power-cancellative semigroup $S$ can be embedded into the smallest uniquely divisible commutative semigroup $T$ in the following sense: If $S$ is embedded into a uniquely divisible semigroup $U$, then $T$ is embedded into $U$.

This was obtained by Hancock [2] and also by the author ([4] or [5]) independently.

For example the additive semigroup $I$ of all positive integers is 
embedded into the additive semigroup $R$ of all positive rational numbers. The following theorem is important.

TheOREM 1. A commutative semigroup $S$ is divisible if and only if there is a set $\Gamma$ such that the annexed product $\tilde{\Pi}_{\alpha \in \Gamma} R_{\alpha}$ is homomorphic onto $S$ where each of $R_{\alpha}$ 's is isomorphic onto the additive semigroup $R$ of all positive rational numbers.

3. Divisible semigroups of degree 1 . If a divisible semigroup $S$ is a homomorphic image of $\widetilde{\Upsilon}_{\alpha \in \Gamma} R_{\alpha}, R_{\alpha} \cong R$, with $|\Gamma|=\lambda$ and it cannot be any homomorphic image of $\tilde{\Pi}_{\alpha \in \Gamma^{\prime}} R_{\alpha}$ with $\left|\Gamma^{\prime}\right|<\lambda$, then $\lambda$ is called the degree of $S$. As a simplest case, we determine all the types of divisible semigroups of degree 1.

THeOREM 2. Let $\xi$ be a non-negative real number, $G$ be a subgroup of the group $R^{\natural}$ of all rational numbers with respect to addition. All congruences on the additive semigroup $R$ of all positive rational numbers are given by the following:

$$
\begin{aligned}
& a \rho_{1} b \quad \text { iff }\left\{\begin{array}{ll}
a=b & a, b \leqq \xi \\
a-b \in G & a, b>\xi
\end{array}\right\}, \quad \xi \text { rational }>0, \\
& a \rho_{2} b \quad \text { iff }\left\{\begin{array}{ll}
a=b & a, b<\xi \\
a-b \in G & a, b \geqq \xi
\end{array}\right\}, \quad \xi \text { rational } \geqq 0, \\
& a \rho_{3} b \quad \text { iff }\left\{\begin{array}{ll}
a=b & a, b<\xi \\
a-b \in G & a, b>\xi
\end{array}\right\}, \quad \xi \text { irrational }>0 .
\end{aligned}
$$

The factor semigroup $R / \rho_{i}$ is denoted by $R_{i}(\xi, G)$. Any divisible semigroup of degree 1 is isomorphic onto $R_{i}(\xi, G) . R_{i}(\xi, G)$ is isomorphic onto $R_{j}(\eta, H)$ if and only if $i=j, \eta \cdot G=\xi \cdot H$, and $G \cong H$.

If $G$ is a cyclic subgroup generated by a rational number $r$, we denote $R_{i}(\xi, G)$ by $R_{i}(\xi, r)$.

4. Minimal divisible subsemigroups. We have the following theorem:

THEOREM 3. Let $S$ be a divisible semigroup and $T$ be a subsemigroup of $S$. Then there is at least one minimal divisible subsemigroup $U$ containing $T . U$ is called minimal if there is no divisible subsemigroup $V$ such that $T \subseteq V \subset U \subset S$.

There may be more than one minimal divisible subsemigroup $U$ containing $T$, but they are not necessarily isomorphic. Accordingly we can say that the analogy to Kulikov's theorem (cf. [1] or [3]) 
in the theory of divisible groups does not hold as the following example shows.

EXAMPLE. Let $R$ be the additive semigroup of all positive rational numbers and let $A=R_{1}(1,3), B=R_{2}(2,3)$; and the natural homomorphisms of $R$ onto $A$ and $B$ due to $\rho_{i}$ are denoted by $\phi$ and $\psi$ respectively. Consider the union $S=A \cup B$ in which we identify elements of $A$ with those of $B$ as follows:

$$
x \phi=y \psi \text { iff } x=y=1 \text { or } x>1, y \geqq 2, x \equiv y(\bmod 3) .
$$

Neither elements of $\{x \phi ; x<1\}$ nor of $\{y \psi ; y \neq 1, y<2\}$ are identified with any other elements. A binary operation in $S$ is defined as follows: Let $a \in A, b \in B$, and let $x \phi=a, y \psi=b$.

$$
a+b=x \phi+y \psi=(x+y) \phi .
$$

Let $C$ be the cyclic subsemigroup generated by $1: C=\{1,2,3,4\}$ with $2=5$. $A$ and $B$ are minimal divisible subsemigroups containing $C$, but $A$ and $B$ are not isomorphic.

5. Embedding of a commutative semigroup into a divisible semigroup. Any commutative semigroup $S$ can be embedded into a commutative divisible semigroup $T$. This was proved by Hancock [2]. We can clarify minimality of $T$ in the following way.

THEOREM 4. Any commutative semigroup $S$ can be embedded into a minimal divisible semigroup $T$ in the sense that $T$ contains no commutative divisible proper subsemigroup $T^{\prime}$ containing $S$. Let $\Im$ be the totality of all nonisomorphic minimal semigroups containing $S . J$ is a complete lattice with respect to the ordering $T_{\alpha} \geqq T_{\beta}$ meaning that $T_{\alpha}$ is homomorphic onto $T_{\beta}$; and hence there are $T_{0}$ and $T_{1}$ belonging to $J$ such that

(5.1) $T_{1}$ is homomorphic onto any $T_{\alpha} \in J$.

(5.2) Every $T_{\alpha} \in J$ is homomorphic onto $T_{0}$.

ExAmple. Let $S$ be a cyclic semigroup $\{1,2,3,4\}$ generated by 1 where $2=5$ is a generating relation. All the minimal divisible semigroups into which $S$ can be embedded are given by the following:

Let $\xi, \eta$ be real numbers $1 \leqq \xi \leqq 2,1 \leqq \eta \leqq 2$.

$T_{1}=R_{2}(2,3), T_{0}=R_{1}(1,3)$, and $R_{i}(\xi, 3)$ where $\xi \neq 1, \xi \neq 2 ; i=1$ or 2 if $\xi$ is rational; $i=3$ if $\xi$ is irrational. Clearly $R_{i}(\xi, 3)$ is isomorphic onto $R_{j}(\eta, 3)$ if and only if $i=j, \xi=\eta$.

6. Remark. The example in $\S 3$ is not only a simplest case but also it plays an important rôle in the general theory of divisible semigroups. A minimal divisible subsemigroup of a divisible semigroup containing an element is of degree 1 . According to Theorem 1, to 
study any divisible semigroup, we need consider all congruences of $\bar{R}=\tilde{\coprod}{ }_{\alpha} R_{\alpha}$. For this purpose the following general result is used: A congruence of a commutative cancellative semigroup $S$ is determined by a system of ideals of $S$ and a system of subgroups of the quotient group of $S$.

\title{
REFERENCES
}

1. L. Fuchs, Abelian groups, Publishing House of the Hungarian Academy of Sciences, Budapest, 1958.

2. V. R. Hancock, Commutative Schreier extensions of semi-groups, Dissertation, Tulane University of Louisiana, New Orleans, La., 1960.

3. L. Ya. Kulikov, On the theory of Abelian groups of arbitrary power, Mat. Sb. (N.S.) 16(58) (1945), 129-162. (Russian)

4. T. Tamura and D. G. Burnell, $A$ note on the extension of semigroups with operators, Proc. Japan Acad. 38 (1962), 495-498.

5. - Extension of groupoids with operators (to appear).

6. T. Tamura, Commutative divisible semigroups (to appear).

University of California, Davis

\section{ALMOST LOCALLY FLAT EMBEDDINGS OF $S^{n-1}$ IN $S^{n}$}

\author{
BY J. C. CANTRELL
}

Communicated by Deane Montgomery, May 7, 1963

1. Introduction. In this paper we use the terminology introduced by Brown in [2]. We consider an $(n-1)$-sphere $S$ embedded in $S^{n}$ and try to determine if the components of $S^{n}-S$ have closures that are $n$-cells (i.e. if $S$ is flat). Brown has shown that if $S$ is locally flat at each of its points, then $S$ is bi-collared [2]. Hence, in this case, $S$ is flat. The principal result of this paper is that if $S$ is not flat in $S^{n}$, $n>3$, and $E$ is the set of points at which $S$ fails to be locally flat, then $E$ contains more than one point. This is a fundamental point at which the embedding problems for $n>3$ differ from those for $n=3$. Throughout this paper we will assume that $n>3$.

2. Outline of proof of principal result. By combining Theorem 1 of [2] and Theorem 2 of [1] one can establish the following.

Lemma 1. Let $S$ be an (n-1)-sphere in $S^{n}$ and $G$ a component of $S^{n}-S$. If $S$ is locally collared in $\mathrm{Cl} G$, then $S$ is collared in $\mathrm{Cl} G$ and $\mathrm{Cl} G$ is an n-cell. 